\title{
Signatures of Dark Matter in Inert Triplet Models
}

\author{
Takeshi $\mathrm{Araki}^{1}$, C. Q. Geng ${ }^{2,3}$ and Keiko I. Nagao ${ }^{2 *}$ \\ ${ }^{1}$ Institute of High Energy Physics, Chinese Academy of Sciences, Beijing 100049, China \\ ${ }^{2}$ Department of Physics, National Tsing Hua University, Hsinchu, Taiwan 300 \\ ${ }^{3}$ National Center for Theoretical Sciences, Hsinchu, Taiwan 300
}

\begin{abstract}
In this talk, we will review the signatures of dark matter in two inert triplet models. For the first model with the hypercharge $\mathrm{Y}=0$, the dark matter mass around $5.5 \mathrm{TeV}$ is favored by both the WMAP data and the direct detection. In contrast, for the second model of $\mathrm{Y}=2$, it is excluded by the direct detection experiments although dark matter with its mass around $2.8 \mathrm{TeV}$ is allowed by WMAP.
\end{abstract}

Keywords: dark matter; inert triplet model.

\section{Introduction}

Since the standard model (SM) cannot give an explanation for dark matter, new physics is expected. In this talk, we review the signatures of dark matter in two models which contain $S U(2)_{L}$ triplet scalars with the hypercharges $\mathrm{Y}=0$ and 2 under $U(1)_{Y}$, respectively 1 . In these models, the triplets are odd under an $Z_{2}$ symmetry so that they neither directly couple to the SM fermions nor develop vacuum expectation values (VEVs), while the neutral components of the triplets are the dark particles. We will refer to the models as the inert triplet models (ITMs). The number of new parameters in the $Y=0$ ITM is three compared to the SM, which is the same as those in the inert singlet model 3 . Clearly, the $Y=0$ ITM is one of the minimal inert models. Similarly, the $Y=2$ ITM like the inert doublet Model $4-6$ has five new parameters. be 2

The relic abundance of the cold dark matter in the universe is determined to

$$
\Omega_{C D M} h^{2}=0.1123 \pm 0.0035
$$

where $h=0.710 \pm 0.025$ is the scaled current Hubble parameter in units of $100 \mathrm{~km} \mathrm{sec}^{-1} \mathrm{Mpc}^{-1}$. On the other hand, the direct searches also provide constraints on dark matter. For example, the spin-independent (SI) dark matter cross section has to satisfy 7

$$
\sigma_{S I} \lesssim\left(5 \times 10^{-44}\right)-10^{-42}
$$

\footnotetext{
*Talk presented by K.I. Nagao at the 2nd International Workshop on Dark Matter, Dark Energy and Matter-Antimatter Asymmetry, Hsinchu, Taiwan, 5-6 Nov 2010.
} 
Note that to have the above constraint, we have assumed that dark matter is the Weakly Interacting Massive Particle (WIMP) with its mass smaller than $O\left(10^{3}\right) \mathrm{GeV}$.

\section{Inert Triplet Models}

\subsection{Inert Triplet Model with $Y=0$}

We extend the SM by adding a zero hypercharge $S U(2)_{L}$ triplet scalar with an unbroken $Z_{2}$ symmetry. The relevant Lagrangian is given by

$$
\begin{aligned}
\mathcal{L} & =\left|D_{\mu} H\right|^{2}+\operatorname{tr}\left|D_{\mu} T\right|^{2}-V(H, T), \\
V(H, T) & =m^{2} H^{\dagger} H+M^{2} \operatorname{tr}\left[T^{2}\right]+\lambda_{1}\left|H^{\dagger} H\right|^{2}+\lambda_{2}\left(\operatorname{tr}\left[T^{2}\right]\right)^{2}+\lambda_{3} H^{\dagger} H \operatorname{tr}\left[T^{2}\right]
\end{aligned}
$$

where $D_{\mu}$ is the covariant derivative including the SM gauge bosons, and the SM Higgs doublet $H$ and the triplet $T$ scalars are defined as

$$
H=\left(\begin{array}{c}
\phi^{+} \\
\frac{1}{\sqrt{2}}(h+i \eta)
\end{array}\right), \quad T=\left(\begin{array}{cc}
\frac{1}{\sqrt{2}} T^{0} & -T^{+} \\
-T^{-} & -\frac{1}{\sqrt{2}} T^{0}
\end{array}\right),
$$

respectively, with $\langle h\rangle=v=246 \mathrm{GeV}$ and $\left\langle T^{0}\right\rangle=0$. The stability condition of the Higgs potential requires

$$
\lambda_{1}, \quad \lambda_{2}>0, \quad 2 \sqrt{\lambda_{1} \lambda_{2}}>\left|\lambda_{3}\right| \text { for negative } \lambda_{3} .
$$

The potential in Eq. (3) becomes a local minimum if and only if

$$
m^{2}<0, \quad 2 M^{2}+\lambda_{3} v^{2}>0,
$$

where $v^{2}=-m^{2} / \lambda_{1}$. After $h$ acquires the VEV, the scalars gain the following masses:

$$
m_{h}^{2}=2 \lambda_{1} v^{2}, m_{T^{0}}^{2}=m_{T^{ \pm}}^{2}=M^{2}+\frac{1}{2} \lambda_{3} v^{2},
$$

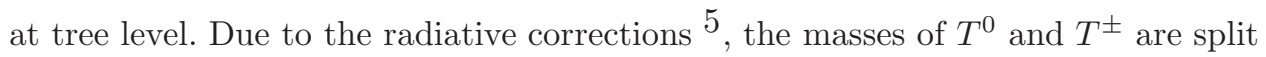
as

$$
m_{T^{ \pm}}=m_{T^{0}}+(166 \mathrm{MeV}) .
$$

Hence, $T^{0}$ turns out to be the lightest component of the triplet scalar with its stability protected by the $Z_{2}$ symmetry.

Since the triplet scalar is added to the SM, one may expect that it affects the so-called oblique ( $\mathrm{S}$ and $\mathrm{T}$ ) parameters. In general, however, an $Y=0$ triplet has no contribution to the $\mathrm{S}$ parameter, while the contribution to the $\mathrm{T}$ parameter is also vanishing in the limit of $m_{T^{0}}=m_{T^{ \pm}}$. Even if we consider the small mass splitting in Eq. (8), its effect is negligibly small, such that

$$
T=\frac{1}{4 \pi c_{w}^{2} s_{w}^{2} m_{Z}^{2}}\left[\left(m_{T^{0}}^{2}+m_{T^{ \pm}}^{2}\right)-\frac{2 m_{T^{0}}^{2} m_{T^{ \pm}}^{2}}{m_{T^{0}}^{2}-m_{T^{ \pm}}^{2}} \log \frac{m_{T^{0}}^{2}}{m_{T^{ \pm}}^{2}}\right] \simeq 0,
$$


where $s_{w}\left(c_{w}\right)$ is the weak mixing angle and $m_{Z}$ is the $Z$ boson mass. Therefore, the constraint on the Higgs boson mass $\left(m_{h}\right)$ from the precision electroweak measurements is the same as that in the SM. In our calculation, we restrict $m_{h}$ to be within the range of

$$
114 \mathrm{GeV}<m_{h}<185 \mathrm{GeV}
$$

as estimated in Ref. 9 with the excluded region of $158 \sim 175 \mathrm{GeV}$ reported by the Tevatron 10 .

\subsection{Inert Triplet Model with $Y=2$}

In the model with the inert triplet scalar of $Y=2$, the $Z_{2}$ invariant scalar potential is given by

$$
\begin{aligned}
V(H, T)= & m^{2} H^{\dagger} H+M^{2} \operatorname{tr}\left[T^{\dagger} T\right]+\lambda_{1}\left|H^{\dagger} H\right|^{2}+\lambda_{2} \operatorname{tr}\left[T^{\dagger} T T^{\dagger} T\right]+\lambda_{3}\left(\operatorname{tr}\left[T^{\dagger} T\right]\right)^{2} \\
& +\lambda_{4} H^{\dagger} H \operatorname{tr}\left[T^{\dagger} T\right]+\lambda_{5} H^{\dagger} T T^{\dagger} H
\end{aligned}
$$

where

$$
T=\left(\begin{array}{cc}
\frac{1}{\sqrt{2}} T^{+} & T^{++} \\
T_{r}^{0}+i T_{i}^{0} & -\frac{1}{\sqrt{2}} T^{+}
\end{array}\right) .
$$

The masses of the scalars are found to be

$$
\begin{aligned}
& m_{h}^{2}=2 \lambda_{1} v^{2}, \\
& m_{T_{r}^{0}}^{2}=m_{T_{i}^{0}}^{2}=M^{2}+\frac{1}{2}\left(\lambda_{4}+\lambda_{5}\right) v^{2}, \\
& m_{T^{ \pm}}^{2}=M^{2}+\frac{1}{2}\left(\lambda_{4}+\frac{\lambda_{5}}{2}\right) v^{2}=m_{T_{r}^{0}\left(T_{i}^{0}\right)}^{2}-\frac{\lambda_{5}}{4} v^{2}, \\
& m_{T^{ \pm \pm}}^{2}=M^{2}+\frac{1}{2} \lambda_{4} v^{2}=m_{T_{r}^{0}\left(T_{i}^{0}\right)}^{2}-\frac{\lambda_{5}}{2} v^{2} .
\end{aligned}
$$

It is clear that $T_{r}^{0}$ can be the lightest $Z_{2}$ odd particle if $\lambda_{5}<0$. Since dark matter must not be a charged particle, we will concentrate on $\lambda_{5}<0$ afterward.

\section{Signatures of Dark Matter}

\subsection{Dark Matter in the model of $Y=0$}

In the model, since the masses of dark matter $\left(T^{0}\right)$ and charged components $\left(T^{ \pm}\right)$ are almost degenerate, the coannihilation effects of $T^{0} T^{ \pm}$and $T^{ \pm} T^{\mp}$ should be included in the calculation of the relic abundance of $T^{0} 11$.

In Figs. 1 and 2 we show the relic abundance of $T^{0}$, where we have used $m i$ crOMEGAS 2.4 12 . For small couplings, i.e. $\lambda_{3} \lesssim 1$, the dark matter annihilation is governed by the weak interaction. So the annihilation cross section does not decrease so much. On the other hand, in the large coupling region (i.e. $\lambda_{3} \gtrsim 1$ ), the main annihilation modes are Higgs interactions. Since the trilinear coupling of $h$ 


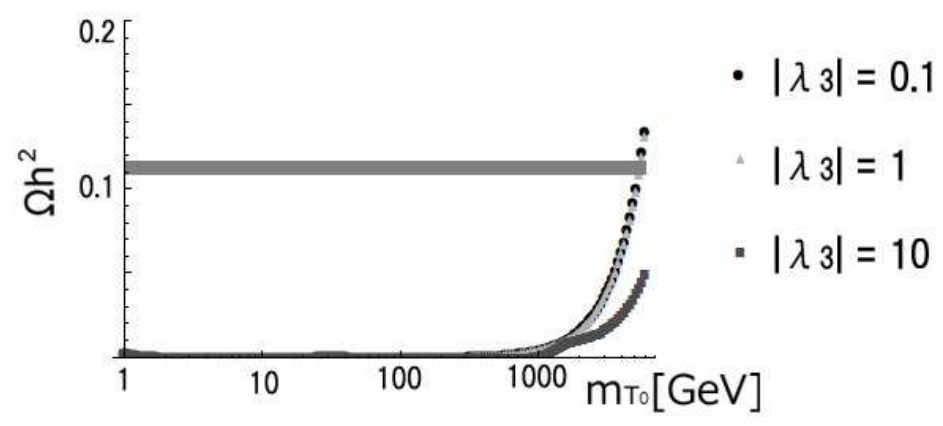

Fig. 1. Relic abundance for $m_{h}=120 \mathrm{GeV}$ with three different choices of $\lambda_{3}$, where the dark gray band represents the region favored by WMAP.

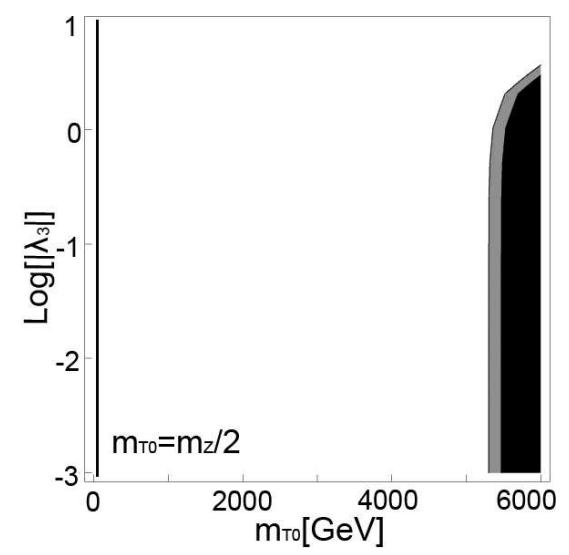

Fig. 2. Relic abundance for $m_{h}=120 \mathrm{GeV}$ with the vertical axis of $\log \left[\lambda_{3}\right]$, where black, gray, and white regions show the parameter regions larger than, agrees with, and smaller than the WMAP constraint, respectively.

involves only $\lambda_{3}$, the cross sections are enhanced by $\lambda_{3}$. From the figure, we find that for $5.4 \mathrm{TeV} \lesssim m_{T^{0}} \lesssim 6 \mathrm{TeV}$, the relic abundance agrees with the WMAP data in Eq. (11).

The SI cross section of the $\mathrm{Y}=0$ ITM is shown in Fig. 3. From the figure, we can see that in most of the region, the model escapes the constraint from the direct search. In this model, the allowed processes are the $T^{0}$-quark $(\mathrm{u}, \mathrm{d})$ scatterings at tree level with the small Yukawa coupling, and $T^{0}$-gluon scatterings at loop level. As a result, the SI cross section is clearly suppressed.

\subsection{Dark Matter in the model of $Y=2$}

The total relic abundance of $T_{r}^{0}$ and $T_{i}^{0}$ is shown in Fig. 5 Note that the masses of $T^{ \pm}$and $T^{ \pm \pm}$are automatically fixed if $m_{T_{r}^{0}}$ and $\lambda_{5}$ are known. Since the degeneracy 


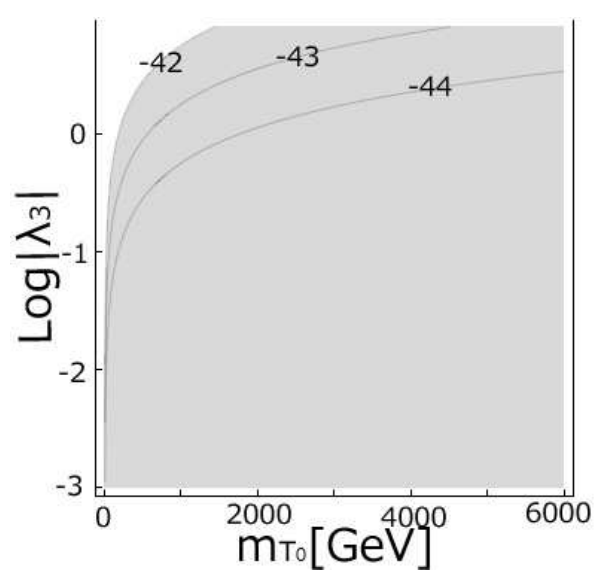

Fig. 3. Spin-independent scattering cross section between the dark matter $T^{0}$ and nucleus particles, where numbers on lines represent the cross sections in $\mathrm{cm}^{2}$ unit, while the light gray region is allowed by direct searches of dark matter.

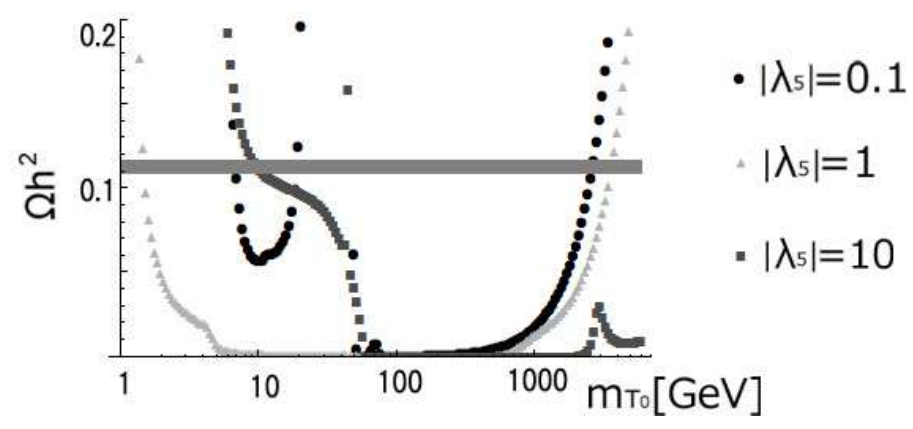

Fig. 4. Relic abundance in the $\mathrm{Y}=2 \mathrm{ITM}$ for $m_{h}=120 \mathrm{GeV}$ with the vertical axis of $\log \left[\left|\lambda_{5}\right|\right]$, where the light gray region is allowed by WMAP.

of triplet masses is lifted at tree level, coannihilations of the triplet scalars are not so effective compared to that in the $Y=0$ case. Thus, the relic abundance gets enhanced. In the large $\left|\lambda_{5}\right|$ region, as the mass degeneracy of the triplet components is lifted, the coannihilation effect becomes weaker than that in the small $\left|\lambda_{5}\right|$, which enhances the relic abundance. However, the annihilation cross section becomes large due to the large couplings of $\lambda_{4}$ and $\left|\lambda_{5}\right|$, which suppresses the relic abundance more effective than the coannihilation effect.

From Fig. 4, one can see that in the small mass region, the relic abundance drastically changes due to the resonance effect as well as the opening of new annihilation final states. In the figure, we have fixed $\lambda_{4}=\left|\lambda_{5}\right| / 8$. As $\lambda_{4}$ approaches to $\left|\lambda_{5}\right|$, the figures become similar to those with small $\left|\lambda_{5}\right|$ since the main interactions enhanced by the large Higgs coupling are proportional to $\left(\lambda_{4}+\lambda_{5}\right)$. In the region 

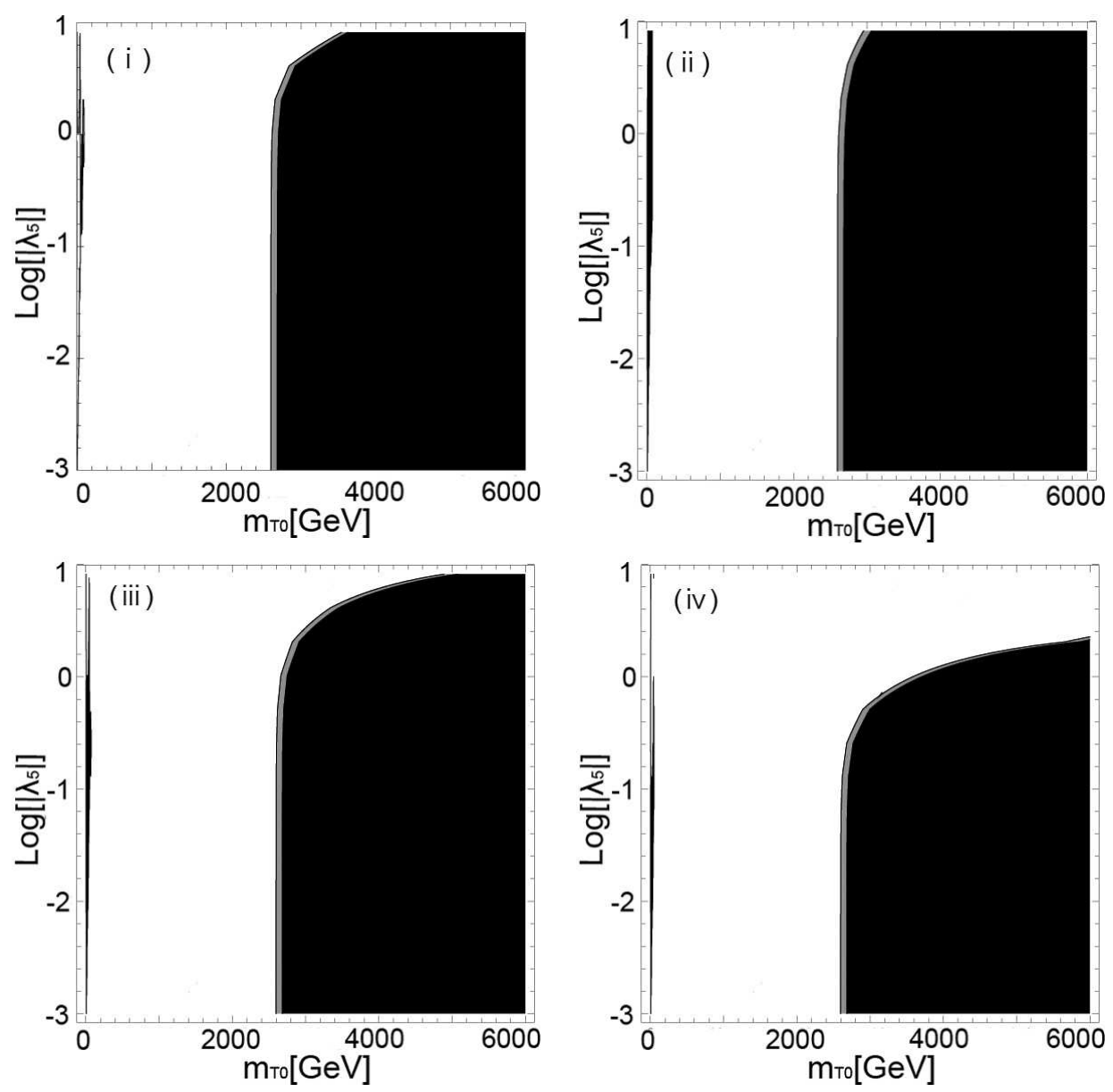

Fig. 5. Legend is same as Fig 2 but for the $Y=2$ inert triplet model, where (i) $\lambda_{4}=\left|\lambda_{5}\right| / 8$, (ii) $\lambda_{4}=\left|\lambda_{5}\right|$, (iii) $\lambda_{4}=2\left|\lambda_{5}\right|$, and (iv) $\lambda_{4}=8\left|\lambda_{5}\right|$.

with $\left|\lambda_{4}\right| /\left|\lambda_{5}\right| \neq 1$, where $T_{r}^{0} T_{r}^{0}\left(T_{i}^{0} T_{i}^{0}\right) \rightarrow h h$ is most effective, the relic abundance is reduced. In the case of $\left|\lambda_{4}\right| /\left|\lambda_{5}\right|=1$, the tri-Higgs couplings proportional to $\left(\lambda_{4}+\lambda_{5}\right)$ are canceled to 0 . Thus, the effective couplings of the Higgs bosons are very weak and the relic abundance is mainly determined by gauge interactions.

We comment on the direct detection of the $Y=2$ case. Unlike those in $Y=0$, there are additional scattering processes in the $\mathrm{Y}=2$ model, which are the $T^{0}$-quark scatterings through the gauge coupling of $T^{0}$ to $\mathrm{Z}$. They have larger cross sections due to the gauge coupling. Because of these large cross sections, almost all region is excluded by the direct detection constraint in Eq. (2). Note that since the scattering process does not depend on the Higgs coupling, this tendency is same even if the ratio of $\lambda_{4}$ and $\left|\lambda_{5}\right|$ changes. 


\section{Conclusion}

We have studied dark matter in the two inert triplet models and we have found that there are allowed regions which agree with the WMAP result in TeVscale for both models. Explicitly,we have shown that in the $\mathrm{Y}=0$ model, the dark matter mass of the neutral scalar around $m_{T^{0}} \sim 5.5 \mathrm{TeV}$ is favored by WMAP, which is also allowed by the direct detection, while in the $\mathrm{Y}=2$ model, $m_{T^{0}} \sim 2.8 \mathrm{TeV}$ is preferred in terms of the relic abundance, but most of the region is excluded by the direct detection constraint since the $T^{0}$-quark scattering cross section mediated by $\mathrm{Z}$ enhanced the SI cross section,

\section{Acknowledgement}

We are grateful to G. Bélanger and A. Pukhov for their kind help for micrOMEGAs. The work of T.A. was supported in part by the National Natural Science Foundation of China under Grant No. 10425522 and No. 10875131. C.Q.G. and K.I.N were partially supported by the National Science Council of Taiwan under Grant No. NSC-98-2112-M-007-008-MY3 and the National Tsing Hua University under the Boost Program No. 97N2309F1.

\section{References}

1. T. Araki, C. Q. Geng and K. I. Nagao, Phys. Rev. D 83, 075014 (2011) arXiv:1102.4906 [hep-ph]].

2. E. Komatsu et al., arXiv:1001.4538 [astro-ph.CO].

3. V. Silveira and A. Zee, Phys. Lett. B 161, 136 (1985); C. P. Burgess, M. Pospelov and T. ter Veldhuis, Nucl. Phys. B 619, 709 (2001); W. L. Guo and Y. L. Wu, JHEP 1010, 083 (2010).

4. E. Ma, Phys. Rev. D 73, 077301 (2006); R. Barbieri, L. J. Hall and V. S. Rychkov, Phys. Rev. D 74, 015007 (2006); L. Lopez Honorez, E. Nezri, J. F. Oliver and M. H. G. Tytgat, JCAP 0702, 028 (2007); E. Lundstrom, M. Gustafsson and J. Edsjo, Phys. Rev. D 79, 035013 (2009); P. Agrawal, E. M. Dolle and C. A. Krenke, Phys. Rev. D 79, 015015 (2009); S. Andreas, M. H. G. Tytgat and Q. Swillens, JCAP 0904, 004 (2009); E. Nezri, M. H. G. Tytgat and G. Vertongen, JCAP 0904, 014 (2009); E. M. Dolle and S. Su, Phys. Rev. D 80, 055012 (2009); C. Arina, F. S. Ling and M. H. G. Tytgat, JCAP 0910, 018 (2009); E. Dolle, X. Miao, S. Su and B. Thomas, Phys. Rev. D 81, 035003 (2010); X. Miao, S. Su and B. Thomas, Phys. Rev. D 82, 035009 (2010); L. Lopez-Honorez and C. E. Yaguna, arXiv:1011.1411 [hep-ph].

5. M. Cirelli and A. Strumia, New J. Phys. 11, 105005 (2009).

6. T. Hambye, F. S. Ling, L. Lopez Honorez and J. Rocher, JHEP 0907, 090 (2009) [Erratum-ibid. 1005, 066 (2010)].

7. J. Angle et al. [XENON Collaboration], Phys. Rev. Lett. 100, 021303 (2008); Z. Ahmed et al. [The CDMS-II Collaboration], Science 327, 1619 (2010).

8. H. H. Zhang, W. B. Yan and X. S. Li, Mod. Phys. Lett. A 23, 637 (2008).

9. LEP Electroweak Working Group, http://lepewwg.web.cern.ch/LEPEWWG/.

10. Tevatron New Phenomena and Higgs Working Group, http://tevnphwg.fnal.gov/

11. K. Griest and D. Seckel, Phys. Rev. D 43, 3191 (1991); S. Mizuta and M. Yamaguchi, Phys. Lett. B 298, 120 (1993). 
8 T. Araki, C.Q. Geng, and K.I. Nagao

12. G. Belanger, F. Boudjema, P. Brun, A. Pukhov, S. Rosier-Lees, P. Salati and A. Semenov, arXiv:1004.1092 [hep-ph].

13. E. J. Chun, JHEP 0912, 055 (2009). 